(2) Open Access Full Text Article

\title{
Impact of smoking on efficacy of PD-I/PD-LI inhibitors in non-small cell lung cancer patients: a meta-analysis
}

This article was published in the following Dove Press journal: OncoTargets and Therapy

\author{
Bingjia Li \\ Xiaoyu Huang \\ Linlin Fu \\ Clinical College, Xuzhou Medical \\ University, Xuzhou, Jiangsu, People's \\ Republic of China
}

Background: Smoking status is associated with the efficacy of non-small cell lung cancer (NSCLC) treatment. Latest clinical trials have depicted the difference in the effectiveness of programmed death-1 (PD-1) and programmed death-ligand 1 (PD-L1) inhibitors in smokers and nonsmokers. However, the lack of statistical power in these trials prevented a final conclusion. The authors postulated that the efficacy of PD-1/PD-L1 inhibitors correlated with smoking status.

Materials and methods: Clinical trials evaluating PD-1 inhibitors versus chemotherapies in smokers and nonsmokers were included. The hazard ratios (HRs) and 95\% confidence intervals (CIs) of overall survival (OS) and progression-free survival (PFS) were used.

Results: A total of 1,981 patients from three Phase III randomized controlled trials (RCTs) were included. PD-1/PD-L1 inhibitors significantly prolonged the OS (HR, 0.69; 95\% CI, 0.60-0.78) and PFS (HR, $0.55 ; 95 \% \mathrm{CI}, 0.43-0.67 ; P=0.027)$ in smoking patients versus chemotherapy. However, among nonsmoking patients, no significant improved OS and PFS were observed compared with chemotherapy.

Conclusion: PD-1 inhibitors were more efficacious in smoking NSCLC patients compared with chemotherapy. No better survival of nonsmoking patients was observed in the treatment of PD-1 inhibitors than chemotherapy. Difference in the efficacy of PD-1 treatment should be taken into consideration in the future guidelines and clinical practice.

Keywords: meta-analysis, PD-1, nivolumab, pembrolizumab, non-small cell lung cancer

\section{Introduction}

More recently, the progress of immune checkpoint inhibitors (ICIs), especially the programmed death-1 (PD-1) inhibitors, is changing the landscape of non-small cell lung cancer (NSCLC) treatment. ${ }^{1,2}$ To date, pembrolizumab and nivolumab have been approved by the US Food and Drug Administration (FDA) in treating melanoma and NSCLC patients. ${ }^{3}$ PD-1 inhibitors, including nivolumab and pembrolizumab, restimulate $\mathrm{T}$ cells which have lost effector and proliferative function. Otherwise, without the PD-1 inhibition, immune cells are likely to lose proliferative function in the tumor microenvironment. PD-L1 is the ligand of PD-1, which was expressed in tumor cells. The tumor cells may escape the antitumor effect of T cells via overexpressing the PD-L1 molecules in the tumor microenvironment. Hence, using ICIs that block the cross talk of PD-L1 molecules and PD-1 molecules may inhibit tumor cells from evading the immunoreaction under this situation. Latest studies reveal that the efficacy of PD-1/ PD-L1 inhibitors may correlate with sex and age; ${ }^{4,5}$ however, little is known whether it is associated with the status of smoking.
Correspondence: Linlin Fu Jiangsu Key Laboratory of Immunology and Metabolism, Department of Pathogenic Biology and Immunology, Xuzhou Medical University, 209 Tongshan Street, Xuzhou, Jiangsu, 221000 , People's Republic of China Email fulinlin_327@I63.com 
A number of studies have evaluated the correlation between smoking and efficacy of lung cancer treatment. ${ }^{6}$ From a preclinical perspective, it was observed that cigarette smoking was associated with therapeutic response to chemotherapy and radiotherapy in cancer cells. ${ }^{7}$ Interestingly, evidence revealed that smoking may induce the epithelialto-mesenchymal transition in lung cancer cell lines. ${ }^{8}$ In the clinical practice, several trials reported that smoking status affected the survival of NSCLC patients in the treatment with anti-PD-1 agents. ${ }^{9}$ However, the lack of statistical power did not generate a meaningful difference. Whether smoking correlates with the effectiveness of PD-1/PD-L1 inhibitors was still unknown. Hence, we hypothesized that the efficacy of nivolumab and pembrolizumab is related to smoking status.

To the best of our knowledge, this is the first study that examined the association between smoking and PD-1/PD-L1 inhibitors' efficacy based on more than 1,000 patients. In this study, we conducted a meta-analysis of 1,981 patients to evaluate the clinical impact of smoking on the effectiveness of PD-1/PD-L1 treatment with a closer look at the survival of NSCLC patients.

\section{Materials and methods}

\section{Study design and search strategy}

A search was performed in Google Scholar, PubMed, and Web of Science until February 2018. The process of search and trial selection was documented as shown in Figure 1. The keywords included PD-1, PD-L1, non-small cell lung cancer, smoke, nivolumab, pembrolizumab, and clinical trials. Articles that were not published in English were excluded. We retrieved all studies of potential conflict of interest.

\section{Selection criteria}

Only trials that met the following five criteria could be included. 1) The trial should enroll NSCLC patients. 2) The intervention should be PD-1/PD-L1 inhibitors. 3) The control group should be other treatments. 4) The outcome of overall survival (OS) or progression-free survival (PFS) of subgroup analysis by smoking status should be reported. 5) The trial

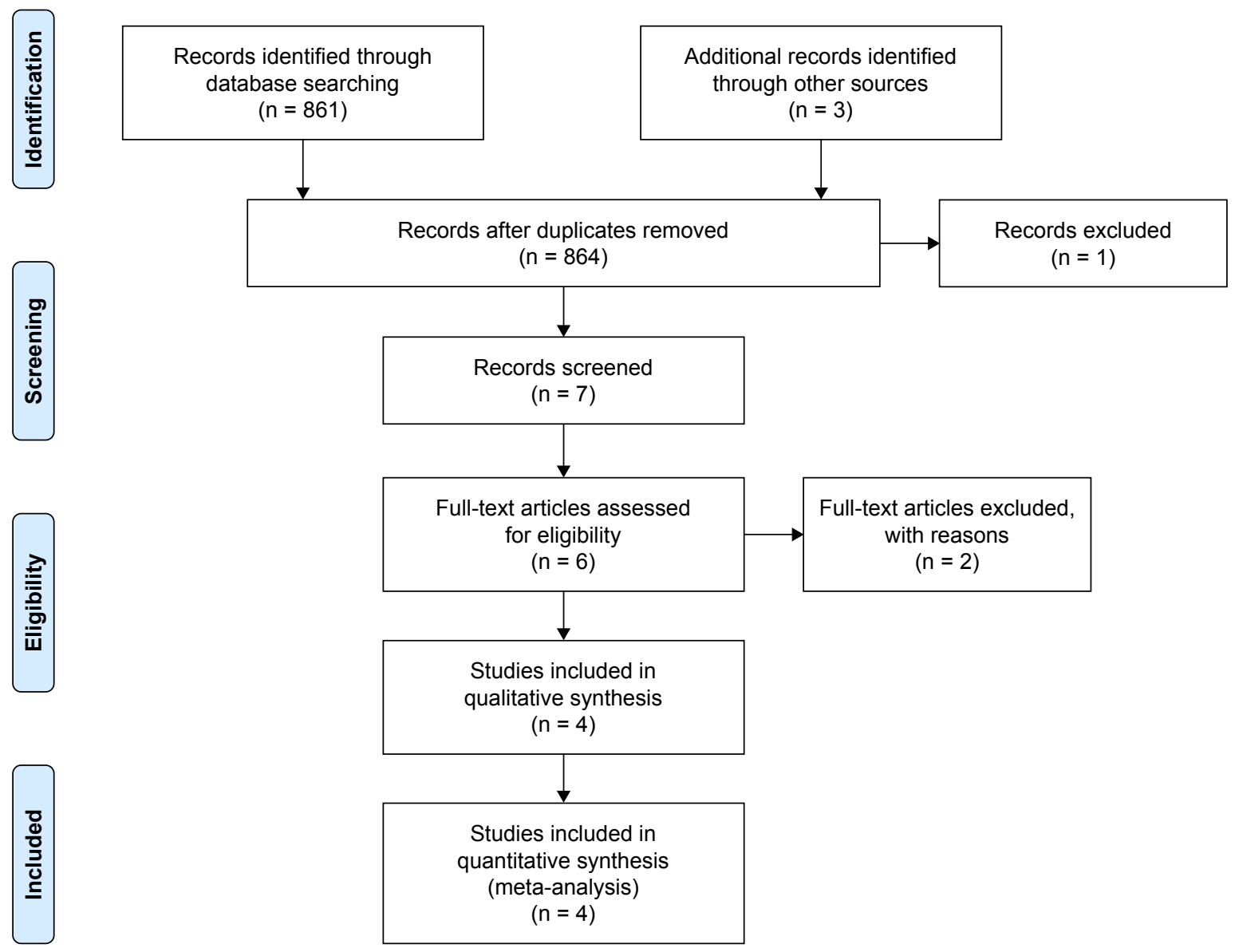

Figure I The procedure for trial selection. 


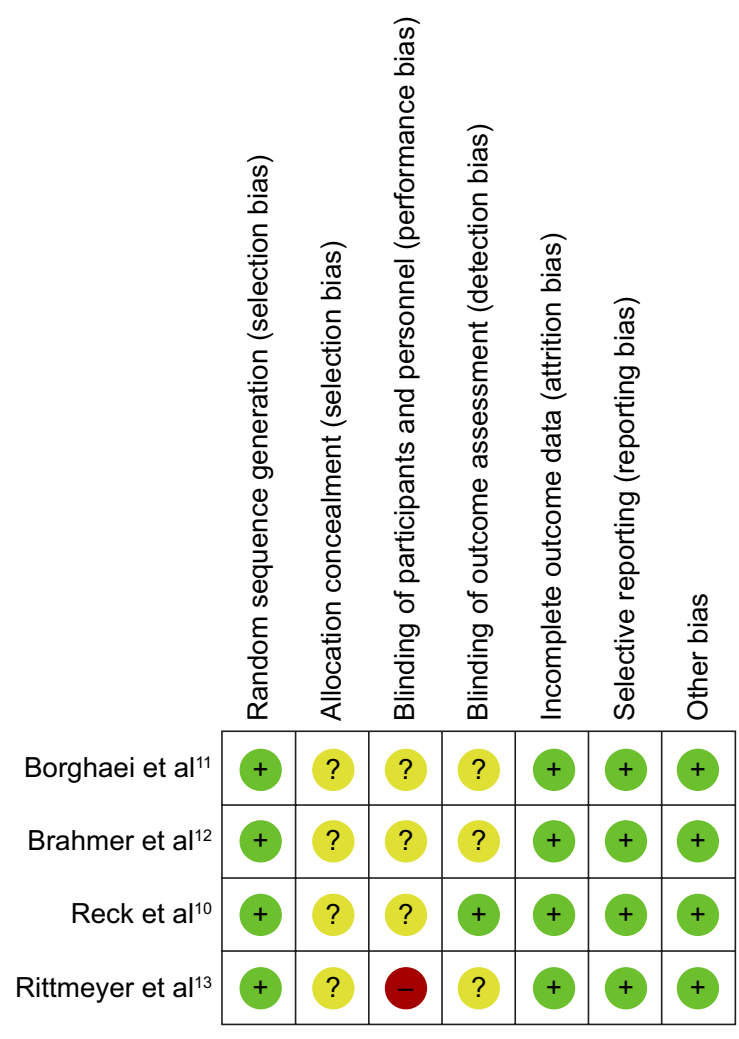

Figure 2 The assessment of risk of bias.

should be Phase III randomized controlled trials (RCTs). Non-English articles should be excluded. In addition, all the duplicated studies were excluded.

\section{Data extraction and risk of bias assessment}

Two authors (Bingjia Li and Xiaoyu Huang) independently extracted the data. We checked the data very carefully. The following information was extracted from the trials: first author, year of publication, number of patients, PD-1/PD-L1 treatments and dosage, hazard ratio (HR) of PFS or OS, the smoking status. Three reviewers (Bingjia Li, Xiaoyu Huang, and Linlin $\mathrm{Fu}$ ) independently assessed the risk of bias using the Cochrane Collaboration tool. Disagreements were solved by the first author (YC). The assessment of risk of bias is shown in Figure 2.

\section{Statistical analyses}

We used Stata 12 for Windows 8.1 to conduct statistical analyses, making forest plots and evaluation of publication bias. Review Manager 5.3 was used to examine the risk of bias. HR and confidence interval (CI) were used as effect sizes. If the $P$-value was $<0.05$, the difference between two arms had a statistical significance. The heterogeneity would be low, moderate, and high, if the $I^{2}$ value was $<25 \%$, $25 \%-50 \%$, and $>50 \%$, respectively. ${ }^{22}$ Publication bias was examined by Egger's test.

\section{Results}

\section{Search results and patient characteristics}

A total of 1,981 patients from four Phase III RCTs were included in this meta-analysis. ${ }^{10-13}$ The procedure for trial selection is shown in Figure 1. All the trials evaluated the efficacy of PD-1/PD-L1 inhibitors, including nivolumab $(\mathrm{n}=826)$, pembrolizumab $(\mathrm{n}=305)$, and atezolizumab $(\mathrm{n}=850)$. All the patients had NSCLC. The characteristics of the RCTs are summarized in Table 1.

\section{OS and PFS}

Three Phase III RCTs were included in OS analysis. ${ }^{11-13}$ Likewise, PFS analysis was based on two Phase III RCTs. ${ }^{10,12}$ There was low or moderate heterogeneity $\left(I^{2}<50 \%\right)$ in OS and PFS analyses, respectively. Hence, fixed-effect models were used in both analyses. One study divided the smoking status into current smoking, former smoking, and never smoking, ${ }^{10}$ and we combined the data of current smoking and former smoking for further analysis.

Our meta-analysis showed that PD-1/PD-L1 inhibitors significantly prolonged the OS in smoking patients (HR, 0.69; 95\% CI, 0.60-0.78) versus chemotherapy (Figure 3). However,

Table I Characteristics of the studies included in the meta-analysis

\begin{tabular}{|c|c|c|c|c|c|}
\hline \multirow[t]{2}{*}{ Study } & \multirow[t]{2}{*}{ Phase } & \multirow[t]{2}{*}{ Cancer } & \multirow[t]{2}{*}{ Arm } & \multicolumn{2}{|c|}{ No. of patients for analysis } \\
\hline & & & & Smokers & Nonsmokers \\
\hline \multirow[t]{2}{*}{ Reck et al ${ }^{10}$} & III, RCT & Advanced NSCLC & Pembrolizumab 200 mg & 149 & 5 \\
\hline & & & ICC & 132 & 19 \\
\hline \multirow[t]{2}{*}{ Borghaei et al" } & III, RCT & Advanced non-squamous NSCLC & Nivolumab 3 mg/kg & 231 & 58 \\
\hline & & & Docetaxel & 227 & 60 \\
\hline \multirow[t]{2}{*}{ Brahmer et $\mathrm{al}^{12}$} & III, RCT & Advanced squamous NSCLC & Nivolumab 3 mg/kg & 121 & $10^{\mathrm{a}}$ \\
\hline & & & Docetaxel & 129 & $7^{\mathrm{a}}$ \\
\hline Rittmeyer et al ${ }^{13}$ & III, RCT & Previous treated NSCLC & $\begin{array}{l}\text { Atezolizumab I,200 mg } \\
\text { Docetaxel }\end{array}$ & 694 & 156 \\
\hline
\end{tabular}

Note: a These subgroups were not included in the analysis because of the small sample size.

Abbreviations: ICC, investigator's choice of chemotherapy; NSCLC, non-small cell lung cancer; RCT, randomized controlled trial. 


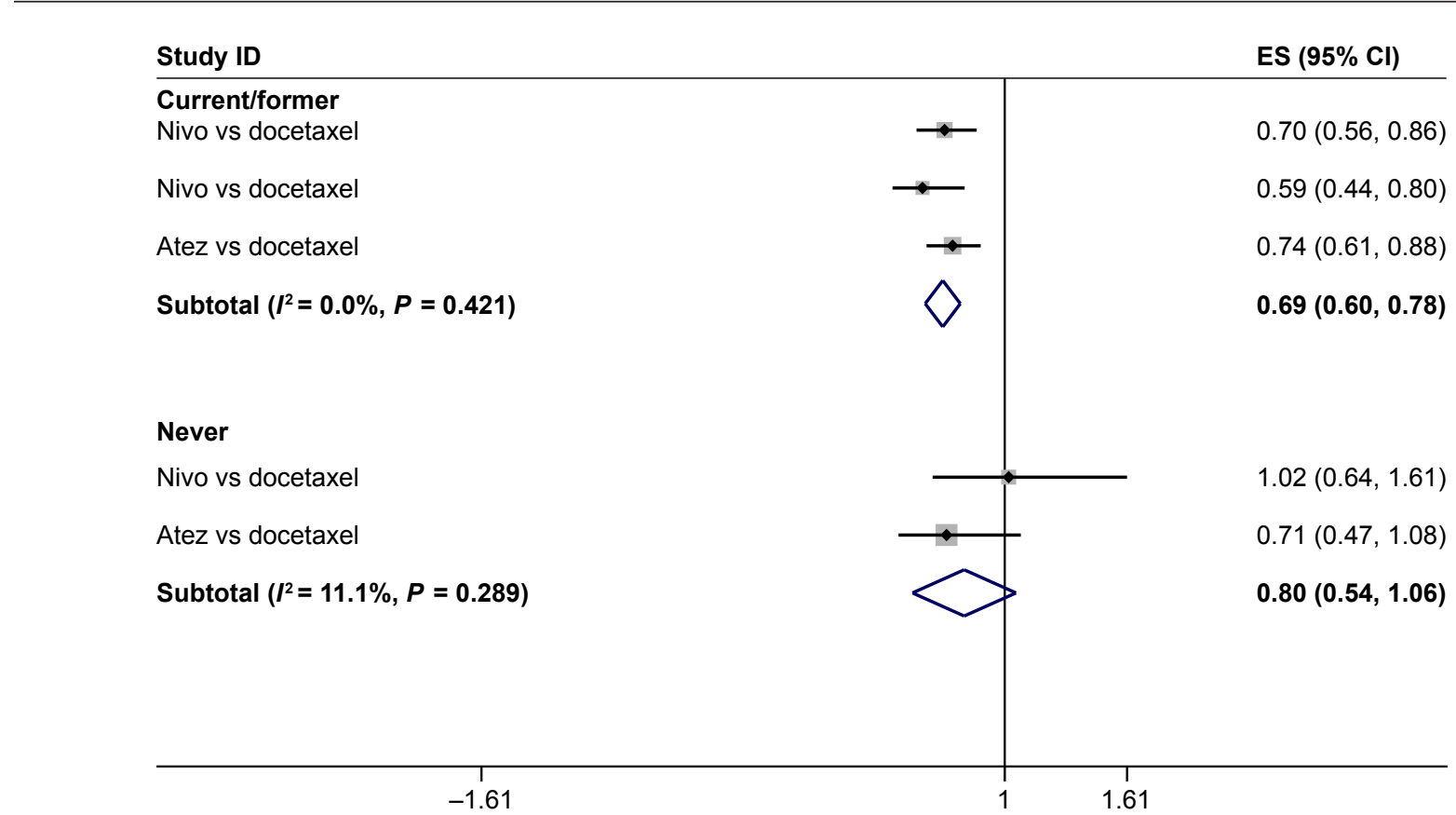

Figure 3 Meta-analysis of OS.

Abbreviations: atez, atezolizumab; $\mathrm{Cl}$, confidence interval; nivo, nivolumab; OS, overall survival.

among nonsmoking patients, no significant improved OS was observed (HR, $0.8 ; 95 \%$ CI, 0.54-1.06; $P>0.05$ ) compared with chemotherapy (Figure 3 ). The PFS analysis indicated a significantly prolonged PFS in smoking patients treated with PD-1 inhibitors (HR, 0.55; 95\% CI, 0.43-0.67; $P=0.027$ ) versus chemotherapy (Figure 4). Similarly, PFS in nonsmoking patients undergoing anti-PD-1 immunotherapy was not significantly longer (HR, $0.90 ; 95 \% \mathrm{CI}, 0.11-7.59 ; P=0.637$ ) than the chemotherapy group.

\section{Publication bias}

No publication bias was observed in both the OS group $(P=0.865)$ and the PFS group $(P=0.635)$ by Egger's test.

\section{Discussion}

To the best of our knowledge, this is the first attempt to collate and evaluate the efficacy of PD-1 inhibitors in smokers and nonsmokers. A few trials have depicted this phenomenon, but the lack of statistical power prevents a final conclusion. ${ }^{9,12}$
Study ID

\section{Current/former}

Pemb vs chemo (Reck et al, 2016) ${ }^{10}$

Nivo vs docetaxel (Borghaei et al, 2015) ${ }^{11}$

Subtotal $\left(I^{2}=23.0 \%, P=0.255\right)$

Never

Pemb vs chemo (Reck et al, 2016) $)^{10}$

Subtotal $\left(I^{2}=\%, P\right)$
ES $(95 \% \mathrm{Cl})$

$0.49(0.33,0.65)$

$0.63(0.47,0.83)$

$0.55(0.43,0.67)$

$0.90(0.11,7.59)$

$0.90(-2.84,4.64)$

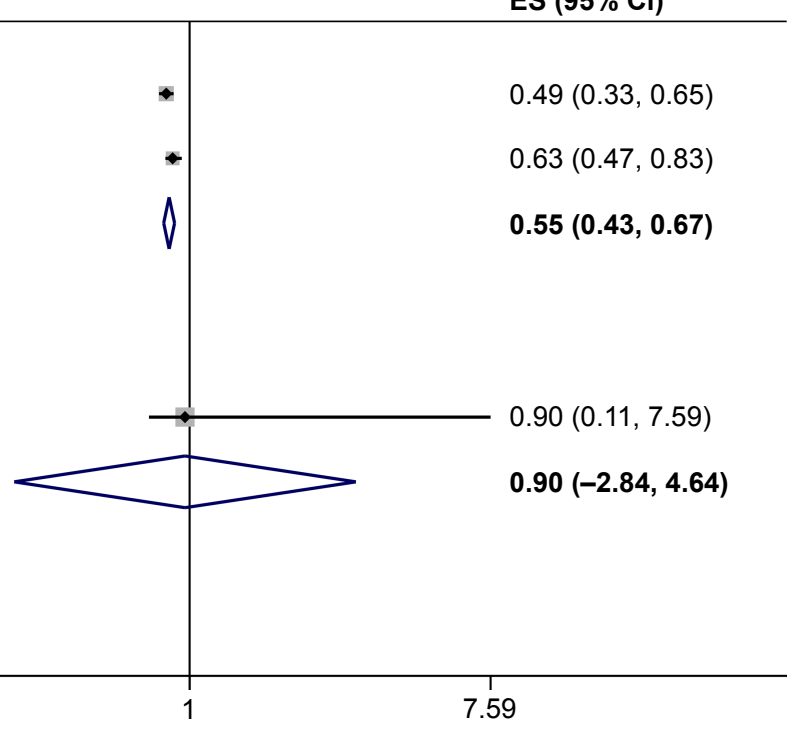


According to our meta-analysis, we found that smokers benefit more from PD-1 inhibitors in both OS and PFS than chemotherapy; however, among nonsmokers, no significantly prolonged survival was found as opposed to controls. This finding was consistent with a recent Phase II trial, ${ }^{14}$ where smoking status was associated with better efficacy of nivolumab. Based on this trial, current/former smokers have a significantly better overall response rate (ORR) than nonsmokers (ORR, 29.1\% versus $4.8 \%$ ) among advanced or recurrent non-squamous NSCLC patients. Better median OS (17.4 versus 14.8 months) and median PFS (2.8 versus 1.4 months) was reported in smokers versus nonsmokers.

Smoking status is associated with the risk of lung cancer, ${ }^{15}$ mortality of lung cancer, ${ }^{16}$ and effectiveness of lung cancer treatment. ${ }^{6}$ According to previous studies, our study offers new evidence in this field. However, the preclinical rationale for our finding still remains unknown. Bavarva et $\mathrm{al}^{17}$ found that $20 \%$ of NSCLC tumors in smokers have mutations in at least one of the MUC4, MUC6 or MUC12 genes in contrast to only $6 \%$ in nonsmokers based on The Cancer Genome Atlas database. Another group demonstrated numbers of long noncoding RNAs (lncRNAs) which may underlie the molecular difference in smokers and nonsmokers. ${ }^{18}$ However, there is still no evidence showing the reasons behind the difference in effectiveness in smokers/nonsmokers. From an immunological perspective, a group reported that smoking significantly regulated the maturation and function of dendritic cells. ${ }^{19}$ Another group proved that smoking will impact the number of Treg cells. ${ }^{20}$ Smoking may also alter the function of natural killer cells and inhibit the antitumor functions. ${ }^{21}$ Despite a direct evidence of the association between smoking, efficacy of PD-1/PD-L1 inhibitor, and immune cells, these findings may partly explain our results. Dialogs among preclinical researchers and clinical scientists are needed to understand the biologic effect of smoking on cancer biology.

An advantage of our study was the ability to assess outcomes in smokers versus nonsmokers in a larger sample size as opposed to data often obtained from comparatively small group in RCTs. Nevertheless, this meta-analysis was faced with several shortcomings. A major concern was that the sample size of nonsmokers was still relatively small, although we had collected all the available data. Also, we cannot retrieve the data of toxicity of PD-1 inhibitors in smokers and nonsmokers. Hence, we cannot conduct analysis of adverse events by smoking status. Further meta-analysis of updated information is required.

\section{Conclusion}

Our meta-analysis showed that PD-1/PD-L1 inhibitors were more efficacious in smoking NSCLC patients compared with chemotherapy. No improvement in survival of nonsmoking patients was observed following treatment with PD-1/PD-L1 inhibitors compared with chemotherapy. Difference in the efficacy of PD-1/PD-L1 treatment should be taken into consideration in the future guidelines and clinical practice.

\section{Acknowledgment}

This study was financially supported by the Top-notch Academic Programs Project of Jiangsu Higher Education Institutions (PPZY2015B161).

\section{Disclosure}

The authors report no conflicts of interest in this work.

\section{References}

1. Queirolo P, Spagnolo F. Atypical responses in patients with advanced melanoma, lung cancer, renal-cell carcinoma and other solid tumors treated with anti-PD-1 drugs: a systematic review. Cancer Treat Rev. 2017;59:71-78.

2. Wu Y, Shi H, Jiang M, et al. The clinical value of combination of immune checkpoint inhibitors in cancer patients: a meta-analysis of efficacy and safety. Int J Cancer. 2017;141(12):2562-2570.

3. Shu CA, Rizvi NA. Into the clinic with nivolumab and pembrolizumab. Oncologist. 2016;21(5):527-528.

4. Wu Y, Ju Q, Jia K, et al. Correlation between sex and efficacy of immune checkpoint inhibitors (PD-1 and CTLA-4 inhibitors). Int J Cancer. Epub 2018 Feb 9.

5. Wu Y, Ju Q, Qian B, Zhang F, Shi H. The effectiveness of PD-1 inhibitors in non-small cell lung cancer (NSCLC) patients of different ages. Oncotarget. 2017;9(8):7942-7948.

6. Condoluci A, Mazzara C, Zoccoli A, Pezzuto A, Tonini G. Impact of smoking on lung cancer treatment effectiveness: a review. Future Oncol. 2016;12(18):2149-2161.

7. Warren GW, Romano M, Sobus S, Balasubramanian S. Abstract 5593 : cigarette smoke and therapeutic response to chemotherapy and radiotherapy in cancer cells. Cancer Res. 2015;75(15 suppl):5593.

8. Liu Y, Luo F, Xu Y, et al. Epithelial-mesenchymal transition and cancer stem cells, mediated by a long non-coding RNA, HOTAIR, are involved in cell malignant transformation induced by cigarette smoke extract. Toxicol Appl Pharmacol. 2015;282(1):9-19.

9. Gettinger S, Rizvi NA, Chow LQ, et al. Nivolumab monotherapy for first-line treatment of advanced non-small-cell lung cancer. J Clin Oncol. 2016;34(25):2980-2987.

10. Reck M, Rodriguez-Abreu D, Robinson AG, et al; KEYNOTE-024 Investigators. Pembrolizumab versus chemotherapy for PD-L1-positive non-small-cell lung cancer. N Engl J Med. 2016;375(19):1823-1833.

11. Borghaei H, Paz-Ares L, Horn L, et al. Nivolumab versus docetaxel in advanced nonsquamous non-small-cell lung cancer. $N$ Engl J Med. 2015;373:1627-1639.

12. Brahmer J, Reckamp KL, Baas P, et al. Nivolumab versus docetaxel in advanced squamous-cell non-small-cell lung cancer. $N$ Engl J Med. 2015;373:123-135.

13. Rittmeyer A, Barlesi F, Waterkamp D, et al; OAK Study Group. Atezolizumab versus docetaxel in patients with previously treated non-small-cell lung cancer (OAK): a phase 3, open-label, multicentre randomised controlled trial. Lancet. 2017;389(10066):255-265.

14. Nishio M, Hida T, Atagi S, et al. Multicentre phase II study of nivolumab in Japanese patients with advanced or recurrent non-squamous nonsmall cell lung cancer. ESMO Open. 2017;1(4):e000108.

15. Zhong L, Goldberg MS, Parent M-É, Hanley JA. Exposure to environmental tobacco smoke and the risk of lung cancer: a meta-analysis. Lung Cancer. 2000;27(1):3-18. 
16. Pope CA 3rd, Burnett RT, Turner MC, et al. Lung cancer and cardiovascular disease mortality associated with ambient air pollution and cigarette smoke: shape of the exposure-response relationships. Environ Health Perspect. 2011;119(11):1616-1621.

17. Bavarva JH, Tae H, McIver L, Garner HR. Nicotine and oxidative stress induced exomic variations are concordant and overrepresented in cancer-associated genes. Oncotarget. 2014;5(13): 4788-4798.

18. Li Y, Wang Z, Nair A, et al. Comprehensive profiling of lincRNAs in lung adenocarcinoma of never smokers reveals their roles in cancer development and prognosis. Genes (Basel). 2017;8(11):321.

19. Givi ME, Folkerts G, Wagenaar GT, Redegeld FA, Mortaz E. Cigarette smoke differentially modulates dendritic cell maturation and function in time. Respir Res. 2015;16:131.
20. Herberth G, Bauer M, Gasch M, et al; Lifestyle and Environmental Factors and Their Influence on Newborns Allergy Risk study Group. Maternal and cord blood miR-223 expression associates with prenatal tobacco smoke exposure and low regulatory T-cell numbers. J Allergy Clin Immunol. 2014;133(2):543-550.

21. Hogan AE, Corrigan MA, O'Reilly V, et al. Cigarette smoke alters the invariant natural killer $\mathrm{T}$ cell function and may inhibit anti-tumor responses. Clin Immunol. 2011;140(3):229-235.

22. DerSimonian R, Laird N. Meta-analysis in clinical trials. Control Clin Trials. 1986;7(3):177-188.

\section{Publish your work in this journal}

OncoTargets and Therapy is an international, peer-reviewed, open access journal focusing on the pathological basis of all cancers, potential targets for therapy and treatment protocols employed to improve the management of cancer patients. The journal also focuses on the impact of management programs and new therapeutic agents and protocols on

\section{Dovepress}

patient perspectives such as quality of life, adherence and satisfaction. The manuscript management system is completely online and includes a very quick and fair peer-review system, which is all easy to use. Visit http://www.dovepress.com/testimonials.php to read real quotes from published authors.

Submit your manuscript here: http://www.dovepress.com/oncotargets-and-therapy-journal 